\title{
Mathematical analysis and numerical simulation of pattern formation under cross-diffusion
}

\author{
Ricardo Ruiz-Baier ${ }^{\mathrm{a}}$, Canrong Tian ${ }^{\mathrm{b}, *}$ \\ ${ }^{a}$ Modeling and Scientific Computing CMCS-MATHICSE-SB, École Polytechnique Fédérale de Lausanne, CH-1015 Lausanne, Switzerland \\ ${ }^{\mathrm{b}}$ Department of Basic Sciences, Yancheng Institute of Technology, Yancheng 224003, China
}

\section{A R T I C L E I N F O}

\section{Article history:}

Received 24 June 2011

Accepted 19 July 2012

\section{Keywords:}

Pattern formation

Cross-diffusion

Pattern selection

Finite volume approximation

\begin{abstract}
A B S T R A C T
Cross-diffusion driven instabilities have gained a considerable attention in the field of population dynamics, mainly due to their ability to predict some important features in the study of the spatial distribution of species in ecological systems. This paper is concerned with some mathematical and numerical aspects of a particular reaction-diffusion system with cross-diffusion, modeling the effect of allelopathy on two plankton species. Based on a stability analysis and a series of numerical simulations performed with a finite volume scheme, we show that the cross-diffusion coefficient plays a important role on the pattern selection.
\end{abstract}

(c) 2012 Elsevier Ltd. All rights reserved.

\section{Introduction}

The theory of spatial pattern generation goes back to the pioneering work of Turing [1] in 1952, where he based the study on a reaction-diffusion model composed by two reactors: an activator and an inhibitor. Essentially, one chemical, the activator, stimulated and enhanced the production of the other chemical, which, in turn, depleted or inhibited the formation of the activator. It was showed that when the diffusion of the inhibitor is greater than that of the activator, the concentration can evolve from the initial near-homogeneity into an inhomogeneous pattern. This implies that the equilibrium of the nonlinear system is asymptotically stable in the absence of diffusion but unstable in the presence of diffusion. This observation was at the time rather counter-intuitive, as one usually may think of diffusion as an homogenizing process.

Spatial patterns in reaction-diffusion systems have attracted the interest of experimentalists and theorists alike during the last few decades. By adding diffusion to a planktonic system, Levin and Segel [2] theoretically demonstrated that diffusion plays an important role in generating spatial patterns. The analytical and numerical methods in [2] have been the routine framework for studying spatial patterns. In [3] the phenomenon of spatial patterns has been shown to actually occur in some closed systems. However, there is still no clear experimental evidence to testify when or how spatial patterns occur under natural biological conditions (see [4] for an extensive review).

Since Levin and Segel's work [2], the concern on diffusion in ecological models has also attracted the attention of biologists. When the movement of the species is combined with population dynamics and multi-species interactions, the resulting governing system is a reaction-diffusion equation of the form

$$
\frac{\partial \mathbf{u}}{\partial t}-\operatorname{div}(\mathscr{D} \nabla \mathbf{u})=\mathbf{G}(\mathbf{u})
$$

\footnotetext{
* Corresponding author. Tel.: +86 051588168591.

E-mail addresses: ricardo.ruiz@epfl.ch (R. Ruiz-Baier),tcr_2001@sohu.com (C. Tian).
} 
where $\mathbf{u}$ is a vector $u_{i}(x, t), i=1,2, \ldots, k$ of species' densities; $D$ is a $k \times k$ matrix of the diffusion coefficients, where the diagonal element is called the self-diffusion coefficient and the non-diagonals are called cross-diffusion coefficients; and $\mathbf{G}$ is the reaction term indicating the interaction between the involved species. Shigesada et al. [5] and Kareiva et al. [6] studied the role of cross-diffusion in the generation of spatial patterns. By now, the model has been extensively studied in the field of ecology, see $[7,8]$ for a review. Several works have been proposed to investigate the existence and uniqueness of weak or global solutions for (1.1) (see [9-12]) and the behavior of stationary states (see e.g. [13-17]). Recently, the pattern formation mechanisms for (1.1) without cross-diffusion have been investigated by [18-24]. Shi et al. [25] showed that cross-diffusion can destabilize or stabilize a uniform equilibrium in a reaction-diffusion system. Recently, cross-diffusion driven Turing instability has been investigated in [26-28]. In addition to these theoretical aspects, an important interest, especially for physicists and biologists, lies in the behavior of numerical approximations exhibiting spatial patterns. In this particular context, there are numerous contributions dealing to some extent with simulations of (1.1), mainly without considering cross-diffusion effects [29-32]. Most of these works propose numerical schemes based on finite difference methods. Although this method allows for a straightforward implementation, the main drawback is that the complexity of cross-diffusion systems induces an extra obstacle in showing convergence of the numerical solutions, at least for classical formulations. More suitable discretization strategies from the viewpoint of numerical analysis are given by finite elements (see for instance [33] for a related problem), and finite volume schemes (see e.g. [34-36]). We adopt the recent finite volume method proposed by Andreianov et al. [34] for the numerical treatment of the underlying reaction-diffusion system with cross-diffusion. By using the linear stability analysis and the finite volume method in [34], we consider a two-species Lotka-Volterra reaction-diffusion competition planktonic system, where each species has an allelopathic effect on the other one, and we show that the cross-diffusion gives rise to the formation of patterns. Moreover, we devote ourselves to the study of some important features of these patterns.

The remainder of this paper is structured as follows. Section 2 gives a precise definition of the mathematical model to be studied, and points to further related work. In Section 3 we deduce from the mathematical standpoint, the role of crossdiffusion in the generation of spatial patterns, and we provide the conditions for these patterns to appear. A finite volume formulation for approximating the governing equations is detailed in Section 4, and some numerical experiments are shown in Section 5, that confirm our theoretical findings, and show that if the cross-diffusion coefficient increases, the selection of spatial patterns converges from the stripes to the spots. We close in Section 6 with some discussions.

\section{The inhibitor-inhibitor model}

In this paper, we consider an inhibitor-inhibitor model, where the reaction kinetics describe a two-plankton-like competition model with allelopathic effects. The nonlinear diffusion coefficients of our model are given by

$$
\mathscr{D}=\left(\begin{array}{cc}
d_{1} & 0 \\
d_{2} d_{3} u_{2} & d_{2}+d_{2} d_{3} u_{1}
\end{array}\right)
$$

and the allelopathic contribution is included in the reaction term G. Specifically, the underlying model consists in the following system:

$$
\left\{\begin{array}{l}
\frac{\partial u_{1}}{\partial t}-d_{1} \Delta u_{1}=u_{1}\left(a_{1}-b_{11} u_{1}-b_{12} u_{2}-e_{1} u_{1} u_{2}\right), \quad(x, t) \in \Omega_{T}, \\
\frac{\partial u_{2}}{\partial t}-d_{2} \Delta\left(u_{2}+d_{3} u_{1} u_{2}\right)=u_{2}\left(a_{2}-b_{21} u_{1}-b_{22} u_{2}-e_{2} u_{1} u_{2}\right), \quad(x, t) \in \Omega_{T}, \\
\frac{\partial u_{1}}{\partial \eta}=\frac{\partial u_{2}}{\partial \eta}=0, \quad(x, t) \in \Sigma_{T}, \\
u_{1}(x, 0)=\psi_{1}(x), \quad u_{2}(x, 0)=\psi_{2}(x), \quad x \in \Omega,
\end{array}\right.
$$

where $\Omega_{T}:=\Omega \times(0, T), \Sigma_{T}:=(\partial \Omega) \times(0, T)$ for a fixed $T>0$. In biological terms, the homogeneous Neumann boundary condition indicates that there is no population flux across the boundary. Here $a_{1}, a_{2}$ are the rates of cell proliferation per hour, $b_{11}$ and $b_{22}$ are the rates of intra-specific competition of the first and the second species, respectively; by $b_{12}$ and $b_{21}$ we denote the rates of inter-specific competition of the first and the second species, respectively, and $a_{i} / b_{i i},(i=1,2)$ are environmental carrying capacities (representing the number of cells per liter). Here $e_{1}$ and $e_{2}$ are the rates of toxic inhibition of the first species by the second and vice versa, respectively. The presence of the nonlinear diffusion term means basically that the disperse direction of $u_{2}$ not only contains the self-diffusion (in which way the species move from a region of high density to a region of low density), but also contains cross-diffusion. More specifically, species $u_{2}$ diffuses with a flux

$$
\mathbf{J}=-\nabla\left(d_{2} u_{2}+d_{2} d_{3} u_{1} u_{2}\right)=-d_{2} d_{3} u_{2} \nabla u_{1}-\left(d_{2}+d_{2} d_{3} u_{1}\right) \nabla u_{2}
$$

Notice that, as $-d_{2} d_{3} u_{2}<0$, the part $-d_{2} d_{3} u_{2} \nabla u_{1}$ of the corresponding flux is directed toward the decreasing population density of the species $u_{1}$.

A universal phenomenon in aquatic ecosystems is that plankton reproduces toxin [37]. A substantial body of literature deals with the construction of models based on differential equations to describe plankton allelopathic interactions inspired 
mainly on field or experimental data (see [38-40] and the references therein). Based on the two species Lotka-Volterra competitive model, Chattopadhyay [39] first proposed differential equations to model plankton allelopathic systems where each species produces a substance toxic to the other, but only when the other species is present. Maynard Smith [40] considered a two species Lotka-Volterra competitive model without diffusion, and studied some stability properties. In terms of (2.2) without diffusion, Mukhopadhyay et al. [41] and Chen et al. [42] studied the effects of time-delay on equilibrium stability and Hopf bifurcation, and additionally Liu et al. [43] investigated the periodicity of positive solutions. Regarding system (2.2) without cross-diffusion, Tian et al. [44-47] considered the stability and periodicity of steady states under Neumann and Dirichlet boundary conditions. However, none of the aforementioned contributions has been able to explain the temporal-spatial periodic fluctuation observed in the experiments reported in [37]. Here is where models considering nonlinear cross-diffusion may provide a more complete description of the mechanisms driving such an interesting phenomenon.

\section{Cross-diffusion driven spatial patterns}

In this section we address some of the conditions for spatial patterns to arise. In particular, we show that when the cross-diffusion is absent the problem (2.2) does not generate spatial patterns, while in the presence of cross-diffusion, the formation of spatial patterns is induced.

\subsection{Linear stability analysis}

Let us start by assuming that the following conditions hold

$$
\frac{b_{12}}{b_{22}}<\frac{a_{1}}{a_{2}}<\frac{b_{11}}{b_{21}} \text { and } \frac{b_{12}}{b_{22}}<\frac{e_{1}}{e_{2}}<\frac{b_{11}}{b_{21}} .
$$

It follows from Lemma 2.1 of [45] that there is a unique positive equilibrium point to system (2.2), denoted by $\mathbf{u}^{*}=\left(u_{1}^{*}, u_{2}^{*}\right)$, where

$$
\begin{aligned}
& u_{i}^{*}=\left(-q_{i j}-\sqrt{q_{i j}^{2}-4 p_{i j} r_{i j}} / 2 p_{i j}\right), \quad i, j=1,2, \\
& p_{i j}=b_{i j} e_{i}-b_{i i} e_{j}, \quad q_{i j}=a_{i} e_{j}-a_{j} e_{i}-b_{i i} b_{j j}+b_{i j} b_{j i}, \quad r_{i j}=a_{i} b_{j j}-a_{j} b_{i j} .
\end{aligned}
$$

In order to study the locally asymptotic stability of the parabolic diffusion equations, we set up the following notation.

Notation 3.1. Let $0=\mu_{1}<\mu_{2}<\cdots \rightarrow \infty$ be the eigenvalues of $-\Delta$ on $\Omega$ under no-flux boundary conditions, and $E\left(\mu_{i}\right)$ be the space of eigenfunctions corresponding to $\mu_{i}$. We define the following space decomposition

(i) $\mathbf{X}_{\mathrm{ij}}:=\left\{\mathbf{c} \cdot \phi_{i j}: \mathbf{c} \in \mathbb{R}^{2}\right\}$, where $\left\{\phi_{i j}\right\}$ is an orthonormal basis of $E\left(\mu_{i}\right)$ for $j=1, \ldots, \operatorname{dim} E\left(\mu_{i}\right)$.

(ii) $\mathbf{X}:=\left\{\mathbf{u} \in\left[C^{1}(\bar{\Omega})\right]^{2}: \frac{\partial u_{1}}{\partial \eta}=\frac{\partial u_{2}}{\partial \eta}=0\right.$ on $\left.\partial \Omega\right\}$, and thus $\mathbf{X}=\bigoplus_{i=1}^{\infty} \mathbf{X}_{\mathbf{i}}$, where $\mathbf{X}_{\mathbf{i}}=\bigoplus_{j=1}^{\operatorname{dim} E\left(\mu_{i}\right)} \mathbf{X}_{\mathbf{i j}}$.

Theorem 3.1. If there is no cross-diffusion, the positive equilibrium point $\mathbf{u}^{*}$ of (2.2) is locally asymptotically stable when (3.1) holds.

Proof. First, for the sake of simplicity, throughout this paper we will denote

$$
\mathbf{G}(\mathbf{u})=\left(\begin{array}{l}
G_{1}(\mathbf{u}) \\
G_{2}(\mathbf{u})
\end{array}\right)=\left(\begin{array}{l}
u_{1} g_{1}(\mathbf{u}) \triangleq u_{1}\left(a_{1}-b_{11} u_{1}-b_{12} u_{2}-e_{1} u_{1} u_{2}\right) \\
u_{2} g_{2}(\mathbf{u}) \triangleq u_{2}\left(a_{2}-b_{21} u_{1}-b_{22} u_{2}-e_{2} u_{1} u_{2}\right)
\end{array}\right) .
$$

The linearization of (2.2) around the state $\mathbf{u}^{*}$ can be therefore expressed by

$$
\mathbf{u}_{\mathbf{t}}=\left(D \Delta+\mathbf{G}_{\mathbf{u}}\left(\mathbf{u}^{*}\right)\right) \mathbf{u},
$$

where $D=\operatorname{diag}\left(d_{1}, d_{2}\right)$, and

$$
\mathbf{G}_{\mathbf{u}}\left(\mathbf{u}^{*}\right)=\left(\begin{array}{ll}
-u_{1}^{*}\left(b_{11}+e_{1} u_{2}^{*}\right) & -u_{1}^{*}\left(b_{12}+e_{1} u_{1}^{*}\right) \\
-u_{2}^{*}\left(b_{21}+e_{2} u_{2}^{*}\right) & -u_{2}^{*}\left(b_{22}+e_{2} u_{1}^{*}\right)
\end{array}\right) \triangleq\left(\begin{array}{ll}
\mathbf{G}_{\mathbf{u} 11} & \mathbf{G}_{\mathbf{u} 12} \\
\mathbf{G}_{\mathbf{u} 21} & \mathbf{G}_{\mathbf{u} 22}
\end{array}\right) .
$$

According to Notation 3.1, the space $\mathbf{X}_{\mathbf{i}}$ is invariant under the operator $D \Delta+\mathbf{G}_{\mathbf{u}}\left(\mathbf{u}^{*}\right)$, and $\lambda$ is an eigenvalue of this operator on $\mathbf{X}_{\mathbf{i}}$, if and only if it is an eigenvalue of the matrix $-\mu_{i} D+\mathbf{G}_{\mathbf{u}}\left(\mathbf{u}^{*}\right)$.

Direct calculation shows that the characteristic polynomial of $-\mu_{i} D+\mathbf{G}_{\mathbf{u}}\left(\mathbf{u}^{*}\right)$ is given by

$$
\psi_{i}(\lambda)=\lambda^{2}-B_{i} \lambda+C_{i}
$$

where

$$
\begin{aligned}
& B_{i}=-u_{1}^{*}\left(b_{11}+e_{1} u_{2}^{*}\right)-u_{2}^{*}\left(b_{22}+e_{2} u_{1}^{*}\right)-d_{1} \mu_{i}-d_{2} \mu_{i}, \\
& C_{i}=\left(u_{1}^{*}\left(b_{11}+e_{1} u_{2}^{*}\right)+d_{1} \mu_{i}\right)\left(u_{2}^{*}\left(b_{22}+e_{2} u_{1}^{*}\right)+d_{2} \mu_{i}\right)-u_{1}^{*} u_{2}^{*}\left(b_{12}+e_{1} u_{1}^{*}\right)\left(b_{21}+e_{2} u_{2}^{*}\right) .
\end{aligned}
$$


Recalling condition (3.1), it is easy to verify that $B_{i}$ and $C_{i}$ are negative. Thus, for each $i \geq 1$, the two roots $\lambda_{i, 1}, \lambda_{i, 2}$ of $\psi_{i}(\lambda)=0$ all have negative real parts, and this concludes the proof.

Theorem 3.1 suggests that under condition (3.1) the self-diffusion cannot induce instabilities that may alter the positive equilibrium point. This statement is consistent with the well-known conclusion that classical competitive models of Lotka-Volterra type cannot generate spatial patterns. Taking cross-diffusion into consideration, we provide the following result.

Theorem 3.2. Assume that condition (3.1) and the following additional condition

$$
u_{1}^{*}\left(b_{11}+e_{1} u_{2}^{*}\right)<u_{2}^{*}\left(b_{12}+e_{1} u_{1}^{*}\right)
$$

holds. If $\mu_{2}<\tilde{\mu}$, then there exists a positive constant $d_{3}^{*}$ such that the equilibrium point $\mathbf{u}^{*}$ of (2.2) is unstable provided that $d_{3} \geq d_{3}^{*}$, where $\mu_{2}$ is given in Notation 3.1 and $\tilde{\mu}$ will be given in (3.5).

Proof. For simplicity, we denote $\boldsymbol{\Phi}(\mathbf{u})=\left(d_{1} u_{1}, d_{2}\left(u_{2}+d_{3} u_{1} u_{2}\right)\right)^{T}$. Linearizing (2.2) around the state $\mathbf{u}^{*}$ yields

$$
\mathbf{u}_{\mathbf{t}}=\left(\boldsymbol{\Phi}_{\mathbf{u}} \Delta+\mathbf{G}_{\mathbf{u}}\left(\mathbf{u}^{*}\right)\right) \mathbf{u}
$$

where

$$
\boldsymbol{\Phi}_{\mathbf{u}}=\left(\begin{array}{cc}
d_{1} & 0 \\
d_{2} d_{3} u_{2}^{*} & d_{2}+d_{2} d_{3} u_{1}^{*}
\end{array}\right) \triangleq\left(\begin{array}{ll}
\boldsymbol{\Phi}_{\mathbf{u} 11} & \boldsymbol{\Phi}_{\mathbf{u} 12} \\
\boldsymbol{\Phi}_{\mathbf{u} 21} & \boldsymbol{\Phi}_{\mathbf{u} 22}
\end{array}\right) .
$$

Direct computation shows that the characteristic polynomial of $-\mu_{i} \Phi_{u}+\mathbf{G}_{\mathbf{u}}\left(\mathbf{u}^{*}\right)$ is

$$
\psi_{i}(\lambda)=\lambda^{2}-\bar{B}_{i} \lambda+\bar{C}_{i}
$$

where

$$
\begin{aligned}
& \bar{B}_{i}=-u_{1}^{*}\left(b_{11}+e_{1} u_{2}^{*}\right)-u_{2}^{*}\left(b_{22}+e_{2} u_{1}^{*}\right)-d_{1} \mu_{i}-d_{2} \mu_{i}, \\
& \bar{C}_{i}=\left(u_{1}^{*}\left(b_{11}+e_{1} u_{2}^{*}\right)+d_{1} \mu_{i}\right)\left(u_{2}^{*}\left(b_{22}+e_{2} u_{1}^{*}\right)+\left(d_{2}+d_{2} d_{3} u_{1}^{*}\right) \mu_{i}\right)-u_{1}^{*}\left(b_{12}+e_{1} u_{1}^{*}\right)\left(u_{2}^{*}\left(b_{21}+e_{2} u_{2}^{*}\right)+d_{2} d_{3} u_{2}^{*} \mu_{i}\right) .
\end{aligned}
$$

Let $\lambda_{1}\left(\mu_{i}\right)$ and $\lambda_{2}\left(\mu_{i}\right)$ be the two roots of $\psi_{i}(\lambda)=0$. We then have

$$
\lambda_{1}\left(\mu_{i}\right)+\lambda_{2}\left(\mu_{i}\right)=B_{i} \text { and } \lambda_{1}\left(\mu_{i}\right) \lambda_{2}\left(\mu_{i}\right)=C_{i} .
$$

In order to ensure that $\operatorname{Re} \lambda_{1}\left(\mu_{i}\right)<0$ and $\operatorname{Re} \lambda_{2}\left(\mu_{i}\right) \geq 0$, a sufficient condition is $\bar{C}_{i}<0$ (based on the fact that $B_{i}<0$ ).

In what follows, we look for conditions so that $\bar{C}_{i}<0$ holds. First, since $\overline{C_{i}}=\operatorname{det}\left(\mu_{i} \boldsymbol{\Phi}_{\mathbf{u}}-\mathbf{G}_{\mathbf{u}}\left(\mathbf{u}^{*}\right)\right)$, it is not difficult to deduce that

$$
\bar{C}_{i}=Q_{2} \mu_{i}^{2}+Q_{1} \mu_{i}+\operatorname{det}\left(\mathbf{G}_{\mathbf{u}}\right),
$$

for

$$
\begin{aligned}
& Q_{2}=d_{1} d_{2}\left(1+d_{3} u_{1}^{*}\right), \\
& Q_{1}=d_{1} u_{2}^{*}\left(b_{22}+e_{2} u_{1}^{*}\right)+d_{2} u_{1}^{*}\left(1+d_{3} u_{1}^{*}\right)\left(b_{11}+e_{1} u_{2}^{*}\right)-d_{2} d_{3} u_{1}^{*} u_{2}^{*}\left(b_{12}+e_{1} u_{1}^{*}\right), \\
& \operatorname{det}\left(\mathbf{G}_{\mathbf{u}}\right)=u_{1}^{*} u_{2}^{*}\left(\left(b_{11}+e_{1} u_{2}^{*}\right)\left(b_{22}+e_{2} u_{1}^{*}\right)-\left(b_{12}+e_{1} u_{1}^{*}\right)\left(b_{21}+e_{2} u_{2}^{*}\right)\right) .
\end{aligned}
$$

Let

$$
\tilde{Q}(\mu)=Q_{2} \mu^{2}+Q_{1} \mu+\operatorname{det}\left(\mathbf{G}_{\mathbf{u}}\right),
$$

and $\tilde{\mu}_{1}$ and $\tilde{\mu}_{2}$ be the two roots of $\tilde{Q}(\mu)=0$ with $\operatorname{Re}\left(\tilde{\mu}_{1}\right) \leq \operatorname{Re}\left(\tilde{\mu}_{2}\right)$. Condition (3.1) implies then, that

$$
\tilde{\mu}_{1}+\tilde{\mu}_{2}=-\frac{Q_{1}}{Q_{2}} \text { and } \tilde{\mu}_{1} \tilde{\mu}_{2}=\frac{\operatorname{det}\left(\mathbf{G}_{\mathbf{u}}\right)}{Q_{2}}>0 .
$$

Furthermore, we have

$$
\begin{aligned}
& \lim _{d_{3} \rightarrow \infty} \frac{Q_{2}}{d_{3}}=d_{1} d_{2} u_{1}^{*}, \\
& \lim _{d_{3} \rightarrow \infty} \frac{Q_{1}}{d_{3}}=d_{2}\left(u_{1}^{*}\right)^{2}\left(b_{11}+e_{1} u_{2}^{*}\right)-d_{2} u_{1}^{*} u_{2}^{*}\left(b_{12}+e_{1} u_{1}^{*}\right), \\
& \lim _{d_{3} \rightarrow \infty} \frac{\tilde{Q}(\mu)}{d_{3}}=\mu\left(d_{1} d_{2} u_{1}^{*} \mu+d_{2}\left(u_{1}^{*}\right)^{2}\left(b_{11}+e_{1} u_{2}^{*}\right)-d_{2} u_{1}^{*} u_{2}^{*}\left(b_{12}+e_{1} u_{1}^{*}\right)\right) .
\end{aligned}
$$


Taking (3.3) in consideration, we see that $\lim _{d_{3} \rightarrow \infty} \frac{\tilde{Q}(\mu)}{d_{3}}=0$ has two real roots, one being zero and the other being positive. A continuity argument allows us to shows that, when $d_{3}$ is large enough, $\tilde{\mu}_{1}$ and $\tilde{\mu}_{2}$ are real and positive, and in addition

$$
\lim _{d_{3} \rightarrow \infty} \tilde{\mu}_{1}=0, \quad \lim _{d_{3} \rightarrow \infty} \tilde{\mu}_{2}=\frac{u_{2}^{*}\left(b_{12}+e_{1} u_{1}^{*}\right)-u_{1}^{*}\left(b_{11}+e_{1} u_{2}^{*}\right)}{d_{1}} \triangleq \tilde{\mu} .
$$

Hence, there exists a positive number $d_{3}^{*}$ such that, when $d_{3} \geq d_{3}^{*}$, the inequalities $\tilde{\mu}<\mu_{2}$, and

$$
\tilde{Q}(\mu)<0 \text { when } \mu \in(0, \tilde{\mu}),
$$

are valid. Since $0<\mu_{2}<\tilde{\mu}$, then $\mu_{2} \in\left(\tilde{\mu}_{1}, \tilde{\mu}_{2}\right)$. It follows that $\tilde{Q}\left(\mu_{2}\right)<0$, which finally implies that $\bar{C}_{i}<0$, which completes the proof.

The above theorems reveal that the cross-diffusion effect is able to destabilize the positive equilibrium point and thereby the solution results in spatial patterns.

\subsection{Turing parameter space}

In view of Theorem 3.2, the fulfillment of the following conditions is sufficient for the positive equilibrium point $\left(u_{1}^{*}, u_{2}^{*}\right)$ being linearly unstable with respect to the particular case of system (2.2):

(i) $\frac{b_{12}}{b_{22}}<\frac{a_{1}}{a_{2}}<\frac{b_{11}}{b_{21}}$ and $\frac{b_{12}}{b_{22}}<\frac{e_{1}}{e_{2}}<\frac{b_{11}}{b_{21}}$.

(ii) $u_{1}^{*}\left(b_{11}+e_{1} u_{2}^{*}\right)<u_{2}^{*}\left(b_{12}+e_{1} u_{1}^{*}\right)$ and $\mu_{2}<\frac{u_{2}^{*}\left(b_{12}+e_{1} u_{1}^{*}\right)-u_{1}^{*}\left(b_{11}+e_{1} u_{2}^{*}\right)}{d_{1}}$.

In this paper, the values satisfying the parameter spaces are taken as follows:

$$
\begin{aligned}
& a_{1}=1, \quad a_{2}=2, \quad b_{11}=0.08, \quad b_{12}=0.06, \quad b_{21}=0.05, \\
& b_{22}=0.15, \quad e_{1}=0.005, \quad e_{2}=0.008, \quad d_{1}=0.1, \quad d_{2}=0.1 .
\end{aligned}
$$

For this particular choice, the positive stationary uniform solution is given by

$$
\left(u_{1}^{*}, u_{2}^{*}\right)=(2.5048,11.0255) .
$$

In Fig. 1 we depict the parameter spaces where instabilities are expected to appear according to conditions (i) and (ii) above. These graphics are obtained by fixing all parameters in (3.6) except for $a_{1}$ and $a_{2}$ (Fig. 1, top left), $b_{11}$ and $b_{12}$ (Fig. 1, top right), $b_{21}$ and $b_{22}$ (Fig. 1, bottom left), and $e_{1}$ and $e_{2}$ (Fig. 1, bottom right).

If no diffusion is considered, problem (2.2) boils down to the dynamical system

$$
\frac{d \mathbf{u}}{d t}=\mathbf{G}(\mathbf{u})
$$

whose phase diagram is presented in Fig. 2. We show computed trajectories for different initial values of $u_{1,0}$ and $u_{2,0}$ which converge to the equilibrium state (3.7), given by the intersection of the (nontrivial) nullclines

$$
G_{1}^{\text {null }}\left(u_{1}\right)=\frac{a_{1}-b_{11} u_{1}}{b_{12}+e_{1} u_{1}}, \quad G_{2}^{\text {null }}\left(u_{1}\right)=\frac{a_{2}-b_{21} u_{1}}{b_{22}+e_{2} u_{1}} .
$$

As the similar method in [8], we are able to calculate the wavenumber explicitly and determine the pattern selection by linearizing cross-diffusion around the stationary uniform solution and taking $d_{3}$ as the Turing bifurcation parameter. From the mathematical viewpoint, the Turing bifurcation occurs when $\operatorname{Im}(\lambda(\mathbf{k}))=0$ and $\operatorname{Re}(\lambda(\mathbf{k}))=0$ at $\mathbf{k}=\mathbf{k}_{\mathbf{c}} \neq 0$, where $\mathbf{k}_{\mathbf{c}}$ is the critical wavenumber and $|\mathbf{k}|^{2}$ is equivalent to the eigenvalue $\mu_{i}$ in Notation 3.1.

Now, replacing $\mu_{i}$ with $|\mathbf{k}|^{2}$, the problem resulting from the linearization of (2.2) around $\mathbf{u}^{*}$ reads

$$
\mathbf{u}_{\mathbf{t}}=\left(\mathbf{G}_{\mathbf{u}}\left(\mathbf{u}^{*}\right)-|\mathbf{k}|^{2} \boldsymbol{\Phi}_{\mathbf{u}}\right) \mathbf{u}
$$

where $\mathbf{G}_{\mathbf{u}}, \boldsymbol{\Phi}_{\mathbf{u}}$ are defined in (3.2) and (3.4). The characteristic equation is

$$
\operatorname{det}\left(\mathbf{G}_{\mathbf{u}}\left(\mathbf{u}^{*}\right)-|\mathbf{k}|^{2} \boldsymbol{\Phi}_{\mathbf{u}}-\lambda E\right)=0 .
$$

In order to find out the critical wavenumber, we only need to confirm that

$$
\min _{|\mathbf{k}|^{2}} \operatorname{det}\left(\mathbf{G}_{\mathbf{u}}\left(\mathbf{u}^{*}\right)-|\mathbf{k}|^{2} \boldsymbol{\Phi}_{\mathbf{u}}\right)=0,
$$

which is a quadratic polynomial of $|\mathbf{k}|^{2}$. In this way, the Turing bifurcation threshold given by $d_{3}^{c}$ satisfies the following equation

$$
4 \operatorname{det}\left(\mathbf{G}_{\mathbf{u}}\left(\mathbf{u}^{*}\right)\right) \operatorname{det}\left(\boldsymbol{\Phi}_{\mathbf{u}}\right)=\left(\mathbf{G}_{\mathbf{u} 11} \boldsymbol{\Phi}_{\mathbf{u} 22}+\mathbf{G}_{\mathbf{u} 22} \boldsymbol{\Phi}_{\mathbf{u} 11}-\mathbf{G}_{\mathbf{u} 12} \boldsymbol{\Phi}_{\mathbf{u} 21}-\mathbf{G}_{\mathbf{u} 21} \boldsymbol{\Phi}_{\mathbf{u} 12}\right)^{2},
$$

and the critical $|\mathbf{k}|^{2}$ assumes the form

$$
\left|\mathbf{k}_{\mathbf{c}}\right|^{2}=\left(\mathbf{G}_{\mathbf{u} 11} \boldsymbol{\Phi}_{\mathbf{u} 22}+\mathbf{G}_{\mathbf{u} 22} \boldsymbol{\Phi}_{\mathbf{u} 11}-\mathbf{G}_{\mathbf{u} 12} \boldsymbol{\Phi}_{\mathbf{u} 21}-\mathbf{G}_{\mathbf{u} 21} \boldsymbol{\Phi}_{\mathbf{u} 12}\right) / 2 \operatorname{det}\left(\boldsymbol{\Phi}_{\mathbf{u}}\right) .
$$



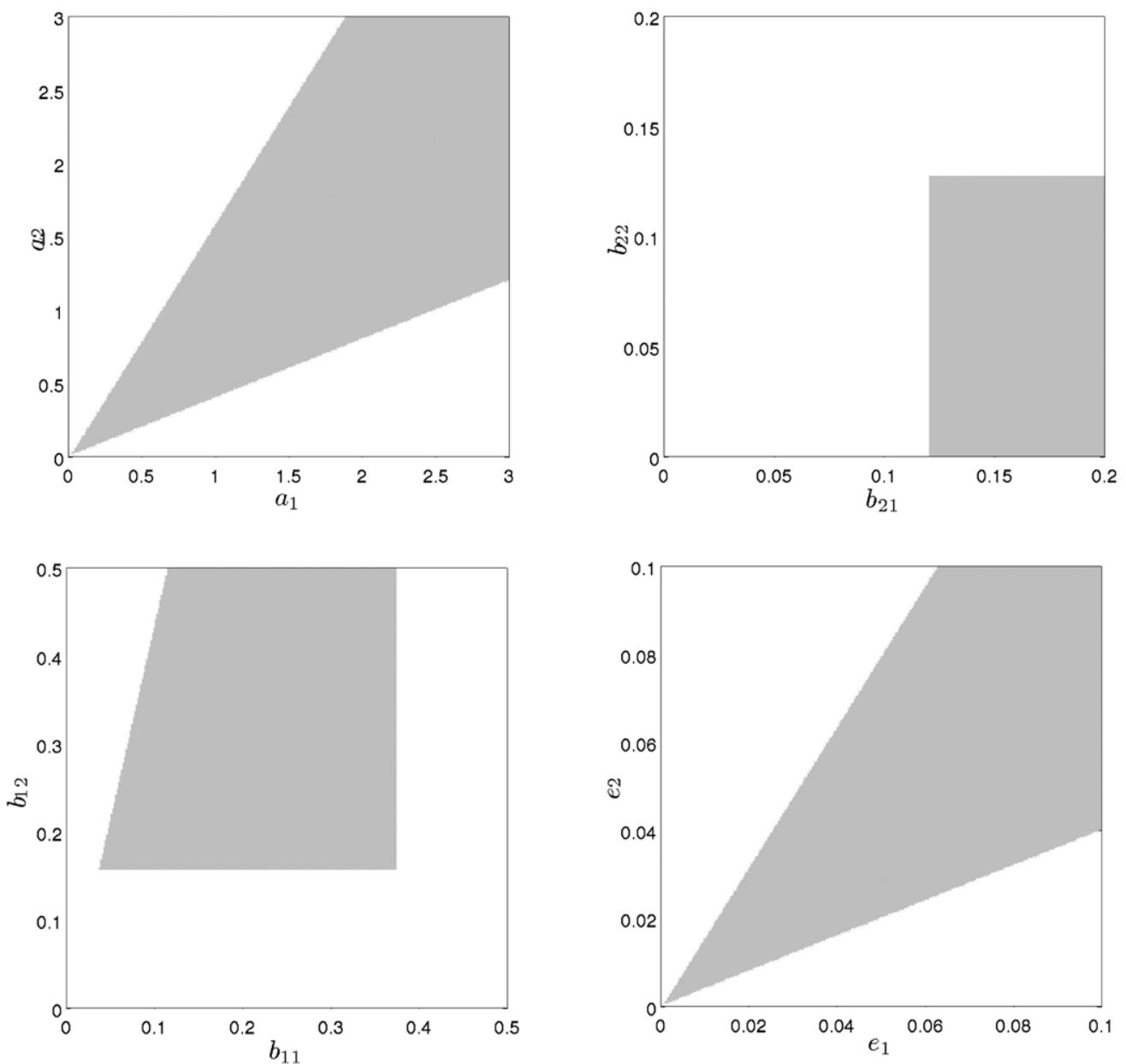

Fig. 1. Turing parameter spaces for model (2.2). Within the gray zones, the solutions remain stable, while for parameters inside the white zones, instabilities are expected to appear.

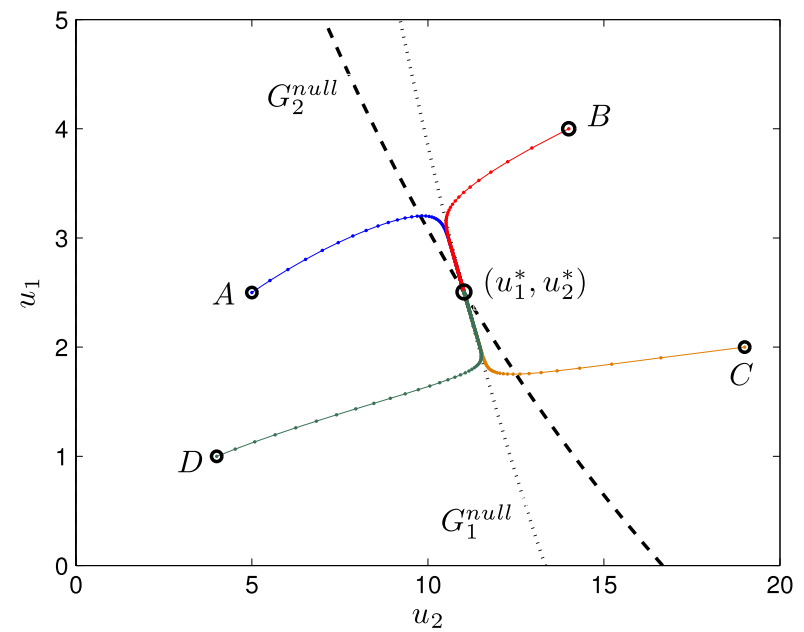

Fig. 2. Phase plane for model (2.2). Four trajectories are represented, starting from the initial states $A=(2.5,5), B=(4,14), C=(2,19), D=(1,4)$ and reaching the intersection of the nullclines $G_{1}^{\text {null }}, G_{2}^{\text {null }}$ of $G_{1}$ and $G_{2}$, respectively. 


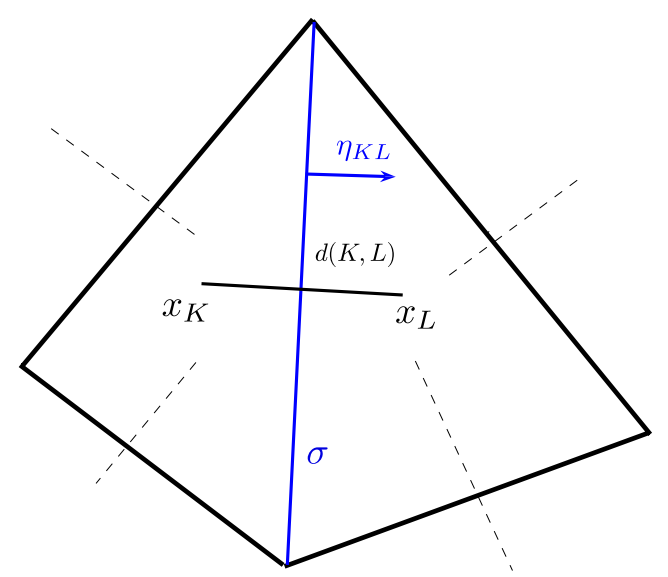

Fig. 3. Sketch of two neighboring control volumes $K$ and $L$ and the orthogonality condition between $\sigma=K \mid L$ and the segment $\overline{x_{K} x_{L}}$ (right).

\section{A finite volume method}

The aim of this section is to present a finite volume scheme, adequate for handling nonlinear reaction-diffusion systems such as (2.2). We start by considering admissible meshes in the sense of [48]. By $\mathcal{T}$ we denote a partition of $\Omega$ into disjoint open and convex polygons $K$ called control volumes of maximum diameter $h$. For a given $K$, we denote by $N(K)$ the set of its neighboring control volumes (sharing a common edge with $K$ ). For a generic $L \in N(K)$, by $d(K, L)$ we denote the distance between $x_{K}$ and $x_{L}$ (the mass centers of $K$ and $L$, respectively) and $\sigma=K \mid L$ is the interface between $K$ and $L$. By $\eta_{K L}$ we denote the normal to $\sigma$, pointing from $K$ to $L$. For all $K \in \mathcal{T}$, we denote by $|K|$ the two-dimensional Lebesgue measure of $K$, while for an interface $\sigma_{K, L},\left|\sigma_{K, L}\right|$ will denote its one-dimensional measure. We have that $\bar{\Omega}=\cup_{K \in \mathcal{T}} \bar{K}, K \cap L=\varnothing$ if $K, L \in \mathcal{T}$ and $K \neq L$, and an orthogonality property is required to hold between the line joining the mass centers of $K, L \in N(K)$, and $\sigma=K \mid L$ (see Fig. 3).

An admissible discretization suitable for obtaining approximate solutions of (2.2) consists of an admissible mesh $\mathcal{T}$ of $\Omega$ and a step size $\Delta t>0$ to discretize the time interval $(0, T)$. We choose $N>0$ as the smallest integer such that $N \Delta t \geq T$, and set $t^{n}:=n \Delta t$ for $n \in\{0, \ldots, N\}$. The discretized allelopathic reaction terms $G_{1, K}^{n+1}, G_{2, K}^{n+1}$ approximate the averages of the components of $\mathbf{G}$ over each control volume

$$
\frac{1}{|K|} \int_{K} G_{1}\left(u_{1}\left(x, t^{n}\right), u_{2}\left(x, t^{n}\right)\right) d x, \quad \frac{1}{|K|} \int_{K} G_{2}\left(u_{1}\left(x, t^{n}\right), u_{2}\left(x, t^{n}\right)\right) d x,
$$

and are given by

$$
G_{1, K}^{n+1}=G_{1}\left(u_{1, K}^{n+1^{+}}, u_{2, K}^{n+1^{+}}\right), \quad G_{2, K}^{n+1}=G_{2}\left(u_{1, K}^{n+1^{+}}, u_{2, K}^{n+1^{+}}\right) .
$$

Here $u_{i, K}^{n+1}$ denote the average of $u_{i} i=1,2$ on a generic space-time element $K \times\left[t^{n}, t^{n+1}\right)$, and the superscript + stands for the positive part of the corresponding quantity.

The classical form of discretizing the components of the diffusion matrix (2.1) consists in taking simply

$$
\mathscr{D}_{i j, K, L}^{n+1}=\mathscr{D}_{i j}\left(\frac{u_{1, K}^{n+1}+u_{1, L}^{n+1}}{2}, \frac{u_{2, K}^{n+1}+u_{2, L}^{n+1}}{2}\right),
$$

where $\mathscr{D}_{i j}$ stands for the $(i, j)$, component of the diffusion matrix, with $i, j=1,2$, and the subscripts $K, L$ denote that these are quantities related to the computation of fluxes through the edge $\sigma=K \mid L$. We have experienced that the treatment of the diffusive terms using instead the following choice [34] (imposed originally to justify the non-negativity of the approximate solutions, and coercivity of the cross-diffusion matrix)

$$
\mathscr{D}_{i j, K, L}^{n+1}:=\mathscr{D}_{i j}\left(\min \left\{u_{1, K}^{n+1^{+}}, u_{1, L}^{n+1^{+}}\right\}, \min \left\{u_{2, K}^{n+1^{+}}, u_{2, L}^{n+1^{+}}\right\}\right),
$$

allows us to obtain equivalent results, but using less restrictive CFL conditions (obviously in the case where a semi-implicit or a fully explicit time discretization is constructed).

The formulation of the finite volume method consists in an $L^{2}$-average of the initial data:

$$
u_{1, K}^{0}=\frac{1}{|K|} \int_{K} u_{1,0}(x) d x, \quad u_{2, K}^{0}=\frac{1}{|K|} \int_{K} u_{2,0}(x) d x,
$$




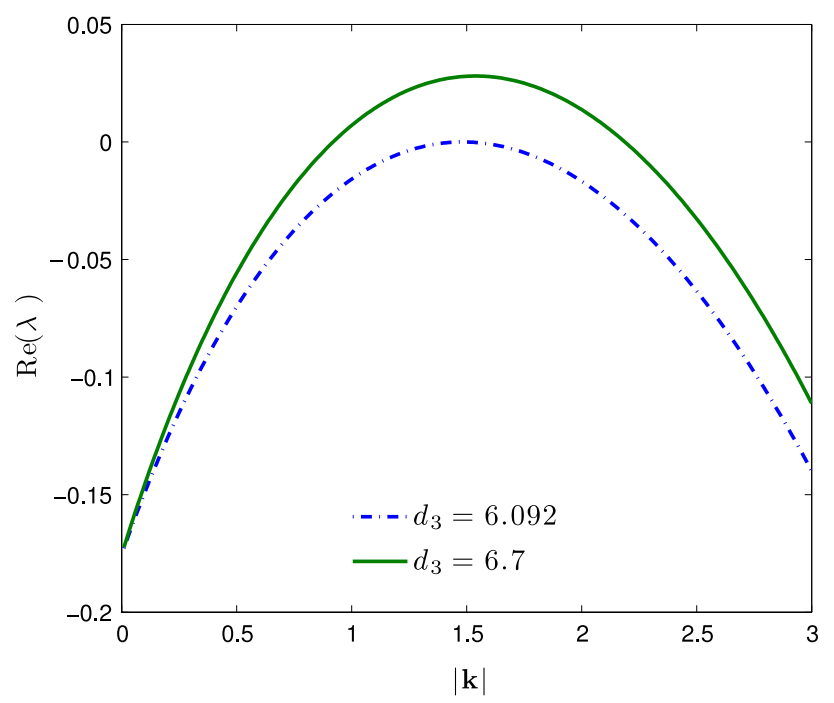

Fig. 4. Dispersion relations for the real part of the eigenvalues, $\operatorname{Re}(\lambda)$ versus the norm of the wave vector $|\mathbf{k}|$.

and the advance in time is achieved by the following implicit scheme. Knowing the numerical solution at time $t^{n}$, determine $\left(u_{1, K}^{n+1}, u_{2, K}^{n+1}\right)$ for every $K \in \mathcal{T}$, such that

$$
\begin{aligned}
& |K| \frac{u_{1, K}^{n+1}-u_{1, K}^{n}}{\Delta t}-d_{1} \sum_{L \in N(K)} \frac{\left|\sigma_{K, L}\right|}{d_{K, L}}\left(u_{1, L}^{n+1}-u_{1, K}^{n+1}\right)=|K| G_{1, K}^{n+1}, \\
& |K| \frac{u_{2, K}^{n+1}-u_{2, K}^{n}}{\Delta t}-\sum_{L \in N(K)} \frac{\left|\sigma_{K, L}\right|}{d_{K, L}}\left[D_{21, K, L}^{n+1}\left(u_{1, L}^{n+1}-u_{1, K}^{n+1}\right)+D_{22, K, L}^{n+1}\left(u_{2, L}^{n+1}-u_{2, K}^{n+1}\right)\right]=|K| G_{2, K}^{n+1} .
\end{aligned}
$$

The homogeneous Neumann boundary conditions are implicitly satisfied in the formulation. The well-posedness of the scheme, positivity-preserving property, and convergence are addressed in detail in [34].

\section{Numerical results}

In this section we present results of computational examples using the finite volume method. The domain of (2.2) is confined to a square domain $\Omega=[0, L] \times[0, L] \subset \mathbb{R}^{2}$. The wavenumber for this two-dimensional domain is thereby

$$
\mathbf{k}=2 \pi(m / L, n / L), \quad|\mathbf{k}|=2 \pi \sqrt{(m / L)^{2}+(n / L)^{2}}, \quad m, n=0,1, \ldots
$$

We set $L=60$, a simple calculation leads to $\mu_{2}=\pi / 60<\frac{u_{2}^{*}\left(b_{12}+e_{1} u_{1}^{*}\right)-u_{1}^{*}\left(b_{11}+e_{1} u_{2}^{*}\right)}{d_{1}}$, which implies that the conditions in Theorem 3.2 are satisfied. According to (3.8) and (3.9), we obtain the Turing bifurcation threshold and the corresponding critical wavenumber

$$
d_{3}^{c}=6.092 \text { and }\left|\mathbf{k}_{\mathbf{c}}\right|^{2}=1.2041 \text {. }
$$

The numerical parameters are set as follows. The domain is discretized using 63,819 control volumes, with a maximum diameter of $h=0.2372$. A time step of $\Delta t=0.1446$ is used. For solving the corresponding nonlinear system arising from the implicit finite volume formulation, we have used a standard Newton method, where at each time step, only a few iterations are required to converge. The linear systems arising from the Newton linearization method are solved by the unsymmetric multi-frontal method (UMFPACK). We do not observe any numerical instabilities during the whole experiments. The initial data is taken as a uniformly distributed random perturbation around the equilibrium state $\left(u_{1}^{*}, u_{2}^{*}\right)$ in $\Omega$, with a variance lower than the amplitude of the final patterns. More precisely,

$$
u_{1}(x, 0)=u_{1}^{*}+\eta_{1}(x), \quad u_{2}(x, 0)=u_{2}^{*}+\eta_{2}(x),
$$

where $\eta_{1} \in[-1.5,1.5]$ and $\eta_{2} \in[-2,2]$.

\subsection{Pattern selection and transient patterns}

For our first test we simulate the selection of different patterns depending on the value of the cross-diffusion coefficient $d_{3}$. In Fig. 4 we depict the real part of the eigenvalues $\operatorname{Re}(\lambda)$ in correspondence with the norm of the wave vector. For the first two rows of Fig. 5 , we have used $d_{3}=6.092$, and we changed this parameter to $d_{3}=6.7$ for the two last rows of Fig. 5 . 

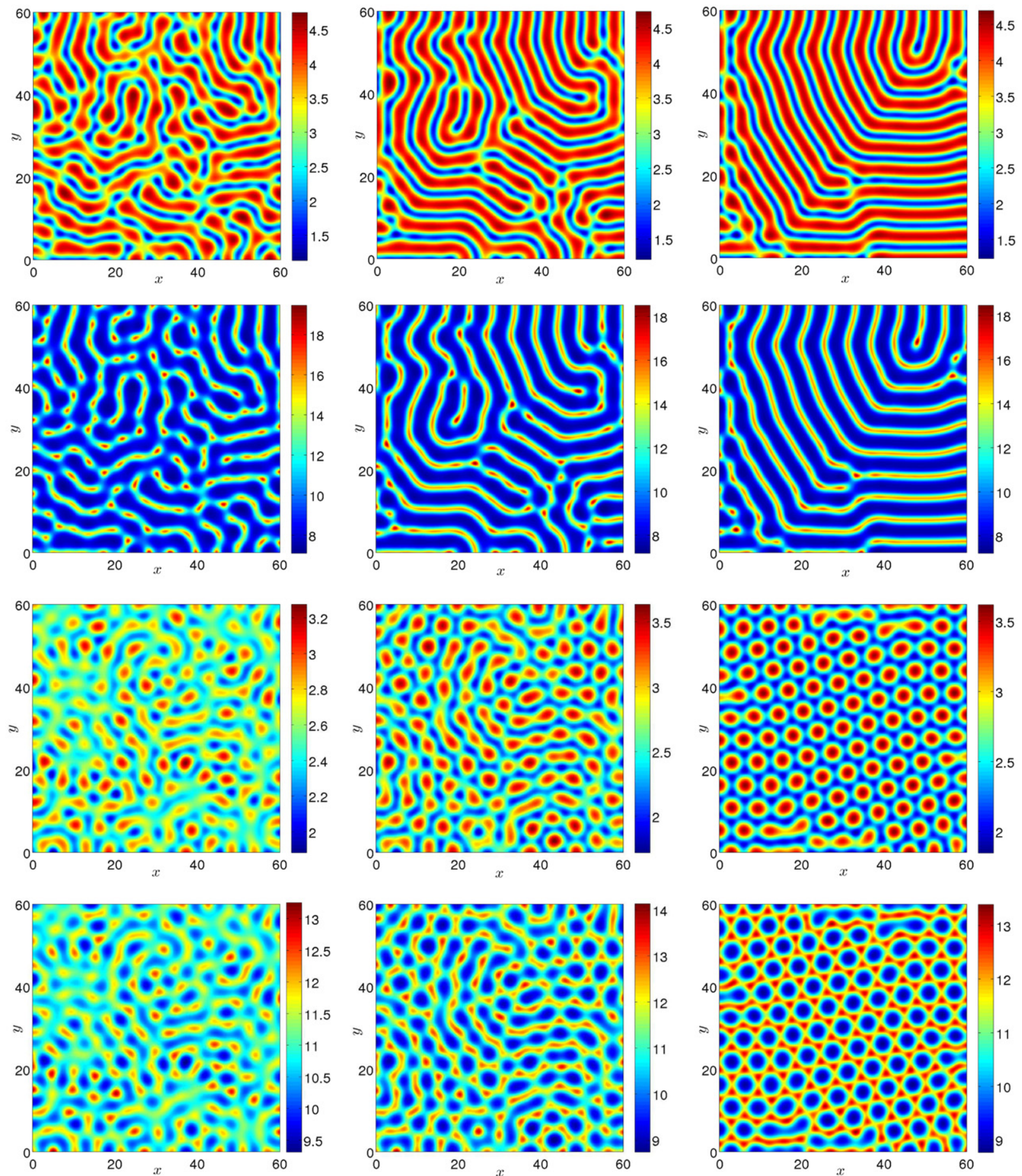

Fig. 5. Example 1. From top to bottom: spatial patterns of species $u_{1}, u_{2}$ for $d_{3}=6.092$, and $u_{1}, u_{2}$ for $d_{3}=6.7$. From left to right: snapshots at time instants $t=200$ (left), $t=500$ (middle) and $t=20000$ (right).

In Fig. 5, the left, middle and right figures are corresponding to the different $d_{3}=200,500$ and 20,000 respectively. Of course, the particular shapes of these patterns are also influenced by the initial data, but we observe a qualitative change in the pattern selection from stripes (top) to spots (bottom). Therefore, we find that when the cross-diffusion coefficient $d_{3}$ increases, the modes of the patterns convert from the stripes to the spots. Thanks to (3.9), we can compute the absolute value of the spatial wavenumber and the spatial wavelength, which are $|\mathbf{k}|=1.2179$ and 5.159 for $d_{3}=6.092$. When $d_{3}=6.7$, the spatial wavenumber and the spatial wavelength are $|\mathbf{k}|=1.2465$ and 5.0407. 


\section{ARTICLE IN PRESS}
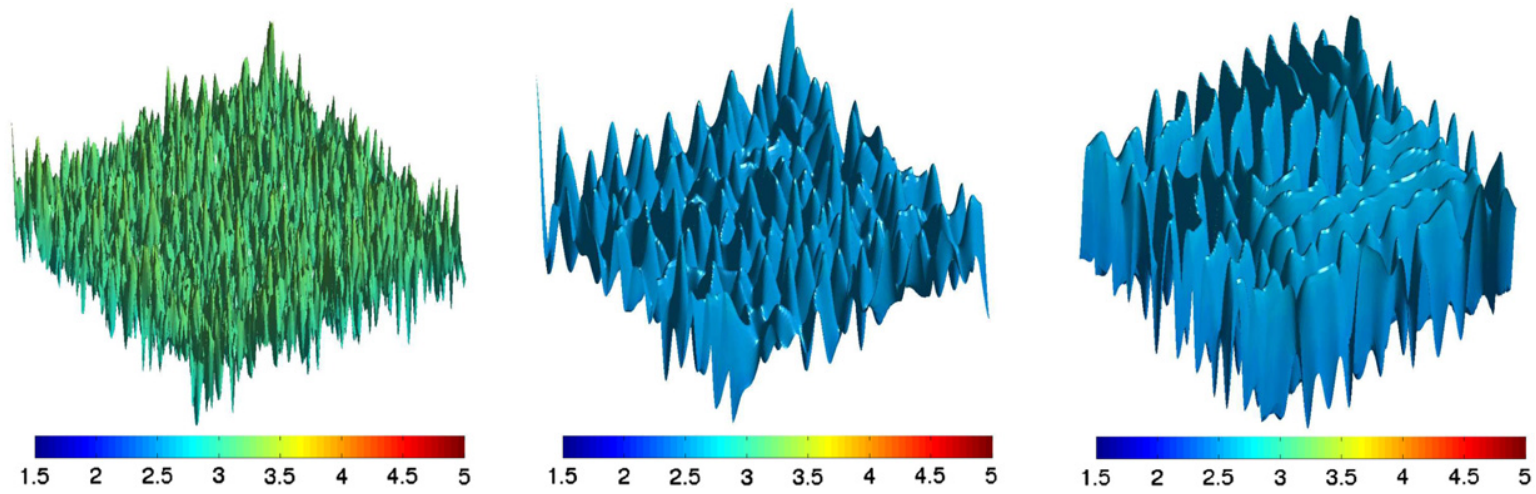

Fig. 6. Spatial patterns of species $u_{1}$ at time instants $t=1, t=50$, and $t=450$.
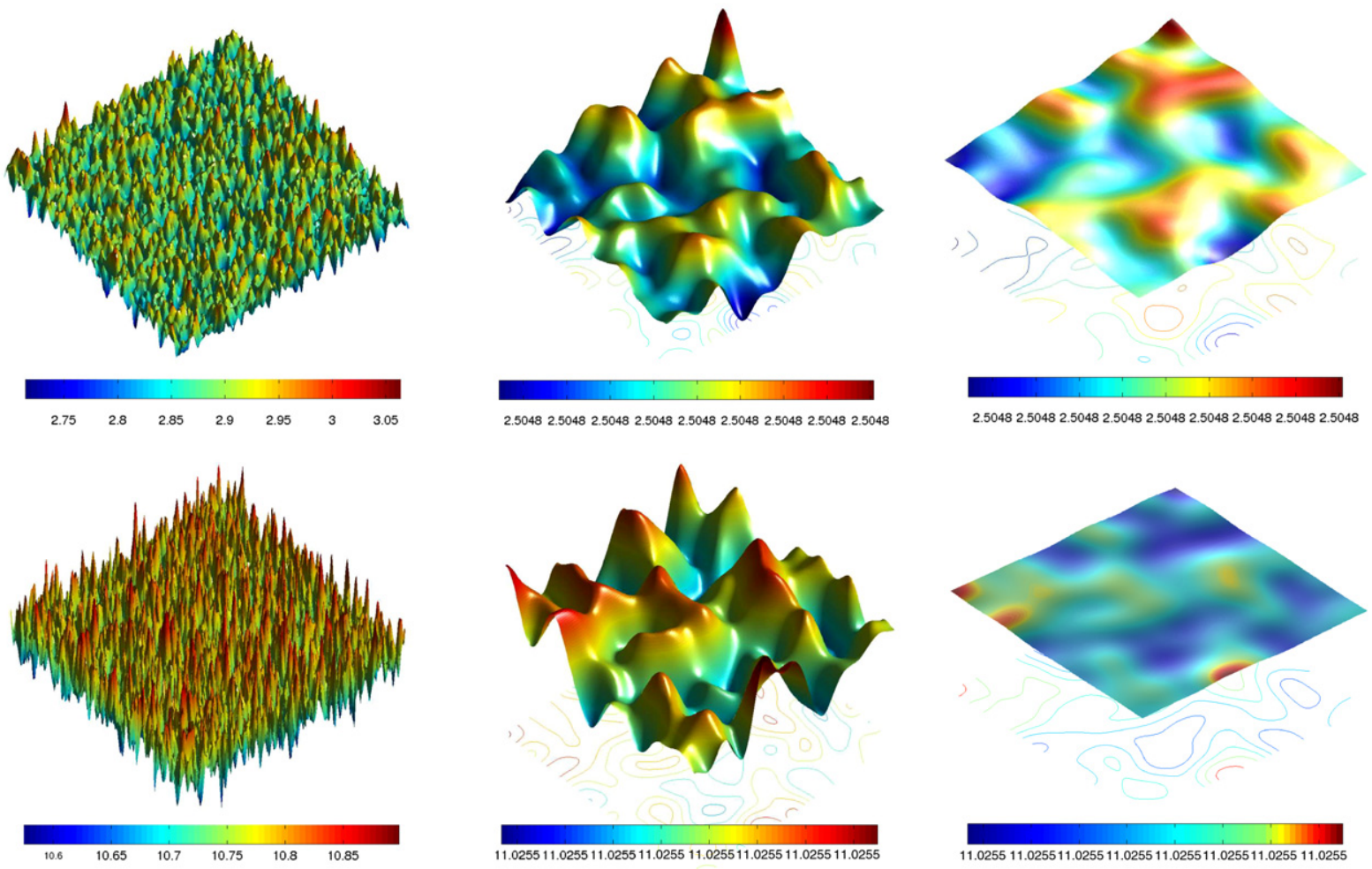

Fig. 7. Example 2. Spatial patterns of species $u_{1}$ (top) and $u_{2}$ (bottom) at time instants $t=1$ (left), $t=50$ (middle) and $t=100$ (right).

Moreover, when $d_{3}=6.092$ and comparing to the figures at the different times, the left figures at $t=200$ are spotted patterns, the middle figures at $t=500$ are transient patterns that present stripes and spots, while the right figures at $t=20,000$ are striped patterns. However, when $d_{3}$ increases to 6.7, transient patterns do not appear. From the two last rows in Fig. 5 we observe only spotted patterns.

In Fig. 6 , we have plotted a different view of snapshots of species $u_{1}$ ( $u_{2}$ follows a similar evolution), where both figures use the same color scale. We readily see that for spatial patterns, their amplitude does not varies in time.

\subsection{Dependence of patterns on the cross-diffusion magnitude}

It is worth pointing out that the variance of the fields $\eta_{1}$, and $\eta_{2}$ in (5.1) plays an important role in the amplitude and formation of spatial patterns. As we have already shown, another key aspect is the magnitude of the cross-diffusion $d_{3}$. For instance, by reducing the effect of cross-diffusion, we do not observe similar spatial patterns; rather, they arrange as larger patches which will eventually smooth out to the constant equilibrium state. This is confirmed by a second numerical example, for which we set $d_{3}=0.1$, while all remaining parameters are taken as in Example 1. Notice in particular the small variance of the colorbars in Fig. 7. We have also found that, as suggested in [12], an alternative strategy for stabilizing 
the system, without the need for decreasing the direct effect of the cross-diffusion, consists in decreasing the value of the inter-specific competition parameters $b_{2 i}, e_{1}$.

\section{Conclusion}

In this paper, we have developed a theoretical framework for studying the phenomenon of pattern formation in a 2D reaction-diffusion system with cross-diffusion. Applying a stability analysis and suitable numerical simulations, we investigate the Turing parameter space, the associated pattern type and a mechanism for pattern selection. The proposed approach has applicability to other reaction-diffusion systems including cross-diffusion, such as chemotaxis, food chain, and cell motility models. In this context, it is of great interest to us the development of a general mathematical and numerical framework that allows for the treatment of certain degenerate quasilinear parabolic systems modeling bacterial growth, that are known to involve several important phenomena such as fractal morphogenesis and branching patterns.

Our results provide clear evidence for the following. (i) Linear stability analysis of homogeneous steady-state solutions provides a reliable predictor of the onset and nature of pattern formation in the reaction-diffusion systems with crossdiffusion. (ii) Turing instabilities occur for successively lower values of the critical cross-diffusion constant $d_{3}^{*}$. (iii) Spatial patterns appear in reaction-diffusion systems with cross-diffusion when the effect of cross-diffusion is larger than a given critical value. (iv) The selection of the spatial pattern transform from stripes to spots when $d_{3}$ increases. (v) The variance of the initial fields plays an important role in the final amplitude and formation of spatial patterns. We have also provided a theoretical support for reaction-diffusion systems with cross-diffusion as viable models for studying Turing pattern formation phenomena.

It is well-known that for a inhibitor-inhibitor system, the formation of patterns does not occur. We introduce the crossdiffusion into the particular aquatic ecological system, and show that this gives rise to Turing-like spatial patterns. All this is confirmed with the help of illustrating numerical simulations that permit us to obtain a qualitative match with the temporal-spatial periodic fluctuation observed in aquatic environments [37].

\section{Acknowledgments}

RR is supported by the European Research Council Advanced Grant "Mathcard, Mathematical Modelling and Simulation of the Cardiovascular System", Project ERC-2008-AdG 227058, and CT is partially supported by the PRC grant NSFC-10801115.

\section{References}

[1] A. Turing, The chemical basis of morphogenesis, Phil. Trans. Royal. Soc. B 237 (1952) 37-72.

[2] S.A. Levin, L.A. Segel, An hypothesis to explain the origin of planktonic patchiness, Nature 259 (1976) 659.

[3] V. Castets, E. Dulos, J. Boissonade, P. De Kepper, Experimental evidence of a sustained standing turing-type nonequilibrium chemical pattern, Phys. Rev. Lett. 64 (1990) 2953-2956.

[4] P.K. Maini, K.J. Painter, H. Nguyen, P. Chau, Spatial pattern formation in chemical and biological system, J. Chem. Soc. Faraday Trans. 93 (1997) $3601-3610$.

[5] N. Shigesada, K. Kawasaki, E. Teramoto, Spatial segregation of interacting species, J. Theor. Biol. 79 (1979) 83-99.

[6] P. Kareiva, G. Odell, Swarms of predators exhibit preytaxis if individual predators use area-restricted, Search. Amer. Natu. 130 (1987) $233-270$.

[7] E.E. Holmes, M.A. Lewis, J.E. Banks, R.R. Veit, Partial differential equations in ecology: spatial interactions and population dynamics, Ecology 75 (1994) $17-29$.

[8] J.D. Murray, Mathematical Biology II: Spatial Models and Biomedical Applications, third ed., Springer-Verlag, New York, 2003.

[9] H. Amann, Dynamic theory of quasilinear parabolic equations II. Reaction-diffusion systems, Differential Integral Equations 3 (1990) 13-75.

[10] W.M. Ni, Diffusion cross-diffusion and their spike layer steady states, in: Notices of the AMS, 1998, pp. 9-18.

[11] L. Chen, A. Jüngel, Analysis of a multi-dimensional parabolic population model with strong cross-diffusion, SIAM J. Math. Anal. 36 (2004) $301-322$.

[12] L. Chen, A. Jüngel, Analysis of a parabolic cross-diffusion population model without self-diffusion, J. Diff. Eqns. 224 (2006) 39-59.

[13] M. Mimura, Y. Nishiura, A. Tesei, T. Tsujikawa, Coexistence problem for two competing species models with density-dependent diffusion, Hiroshima Math. J. 14 (1984) 425-449.

[14] Y. Lou, W.M. Ni, Diffusion, self-diffusion and cross-diffusion, J. Diff. Eqns. 131 (1996) 79-131.

[15] K. Kuto, Stability of steady-state solutions to a prey-predator system with cross-diffusion, J. Diff. Eqns. 197 (2004) 293-314.

[16] M. Wang, Stationary patterns for a prey-predator model with prey-dependent and ratio-dependent functional responses and diffusion, Physica D 196 (2004) $172-192$.

[17] R. Peng, Qualitative analysis of steady states to the Sel'kov model, J. Diff. Eqns. 241 (2007) 386-398.

[18] J.J. Jiao, L.S. Chen, J.J. Nieto, T. Angela, Permanence and global attractivity of stage-structured predator-prey model with continuous harvesting on predator and impulsive stocking on prey, Appl. Math. Mech. -Engl. Ed. 29 (2008) 653-663.

[19] B.I. Camara, Waves analysis and spatiotemporal pattern formation of an ecosystem model, Nonl. Anal. RWA 12 (2011) $2511-2528$.

[20] G.Z. Zeng, F.Y. Wang, J.J. Nieto, Complexity of a delayed predator-prey model with impulsive harvest and holling type II functional response, Adv. Complex Syst. 11 (2008) 77-97.

[21] J.A.L. Silva, J.A. Barrionuevo, F.T. Giordani, Synchronism in population networks with non linear coupling, Nonl. Anal. RWA 11 (2010) $1005-1016$.

[22] L.M. Wang, L.S. Chen, J.J. Nieto, The dynamics of an epidemic model for pest control with impulsive effect, Nonl. Anal. RWA 11 (2010) $1374-1386$.

[23] X.N. Guan, W.M. Wang, Y.Y. Cai, Spatiotemporal dynamics of a Leslie-Gower predator-prey model incorporating a prey refuge, Nonl. Anal. RWA 12 (2011) 2385-2395.

[24] W.B. Wang, J.H. Shen, J.J. Nieto, Permanence and periodic solution of predator-prey system with holling type functional response and impulses, Discrete Dyn. Nat. Soc. 1 (2007) 81756.

[25] J.P. Shi, Z.F. Xie, K. Little, Cross-diffusion induced instability and stability in reaction-diffusion systems, J. Appl. Anal. Comput. 1 (2011) $95-119$.

[26] C. Tian, Z. Ling, Z. Lin, Turing pattern formation in a predator-prey-mutualist system, Nonl. Anal. RWA 12 (2011) 3224-3237.

[27] G. Gambino, M.C. Lombardo, M. Sammartino, Turing instability and traveling fronts for a nonlinear reaction-diffusion system with cross-diffusion, Math. Comput. Simul. $82(2012)$ 1112-1132.

[28] A. El Hamidi, M. Garbey, N. Ali, On nonlinear coupled diffusions in competition systems, Nonl. Anal. RWA 13 (2012) $1306-1318$. 
[29] R.A. Barrio, C. Varea, J.L. Aragón, A two-dimensional numerical study of spatial pattern formation in interacting turing systems, Bull. Math. Biol. 61 (1999) 483-505.

[30] M.A.J. Chaplain, M. Ganesh, I.G. Graham, Spatio-temporal pattern formation on spherical surfaces: numerical simulation and application to solid tumor growth, J. Math. Biol. 42 (2001) 387-423.

[31] S.S. Liaw, C.C. Yang, R.T. Liu, Turing model for patterns of lady beetles, Phys Rev. E. 64 (2001) 041909.

[32] E.J. Crampin, W.W. Hackborn, P.K. Maini, Pattern formation in reaction-diffusion models with nonuniform domain growth, Bull. Math. Biol. 64 (2002) 746-769.

[33] J.W. Barrett, J.F. Blowey, Finite element approximation of a nonlinear cross-diffusion population model, Numer. Math. 98 (2004) $195-221$.

[34] B. Andreianov, M. Bendahmane, R. Ruiz-Baier, Analysis of a finite volume method for a cross-diffusion model in population dynamics, M3AS Math. Models Meth. Appl. Sci. 21 (2011) 307-344.

[35] S. Berres, R. Ruiz-Baier, A fully adaptive numerical approximation for a two-dimensional epidemic model with nonlinear cross-diffusion, Nonl. Anal. RWA 12 (2011) 2888-2903.

[36] S. Berres, R. Ruiz-Baier, H. Schwandt, E. Tory, An adaptive finite-volume method for a model of two-phase pedestrian flow, Netw. Het. Media 6 (2011) 401-423.

[37] E.L. Rice, Allelopathy, second ed., Academic Press, New York, 1984.

[38] E.M. Gross, Allelopathy of aquatic autotrophs, Critical Reviews in Plant Science 22 (2003) 313-339.

[39] J. Chattopadhyay, Effects of toxic substances on a two-species competitive system, Ecol. Model. 84 (1996) 287-289.

[40] J. Maynard Smith, Models in Ecology, Cambridge University, 1974.

[41] A. Mukhopadhyay, J. Chattopadhyay, P.K. Tapaswi, A delay differential equations model of plankton allelopathy, Math. Biosci. 149 (1998) 167-189.

[42] F.D. Chen, Z. Li, X.X. Chen, J. Laitochova, Dynamic behaviors of a delay differential equation model of plankton allelopathy, J. Comput. Appl. Math. 206 (2007) 733-754.

[43] Z.J. Liu, L.S. Chen, Positive periodic solution of a general discrete non-autonomous difference system of plankton allelopathy with delays, J. Comput. Appl. Math. 197 (2006) 446-456.

[44] C. Tian, Z. Lin, Periodic solutions of reaction diffusion systems in a half-space domain, Nonl. Anal. RWA 9 (2008) $811-821$.

[45] C. Tian, L. Zhang, Z. Ling, The stability of a diffusion model of plankton allelopathy with spatio-temporal delays, Nonl. Anal. RWA 10 (2009) 2036-2046.

[46] C. Tian, Z. Lin, Asymptotic behavior of solutions of a periodic diffusion system of plankton allelopathy, Nonl. Anal. RWA 11 (2010) 1581-1588.

[47] C. Tian, Coexistence and asymptotic periodicity in a competition model of plankton allelopathy, Acta Appl. Math. 113 (2011) $195-206$.

[48] R. Eymard, T. Gallouët, R. Herbin, Finite volume methods, in: P.G. Ciarlet, J.L. Lions (Eds.), Handbook of Numerical Analysis, Vol. VII, North-Holland, Amsterdam, 2000, pp. 713-1020. 